\title{
Le Château Cugny, un escarpement particulièrement gélif du Jura
}

"It is not possible for one person to follow the actual slope development in detail over wide areas and long periods. This type of slope studies therefore demands contributions from many geomorphologists working in various environments, before safe generalizations can be made." A. Rapp (1961, p. 11).

\section{Introduction:}

L'escarpement rocheux de Château Cugny $(564,9 / 236,9)$ est situé à environ $6 \mathrm{~km}$ au $\mathrm{NW}$ de Saignelégier à mi-chemin entre cette localité et le Doubs. Formé de roches gélives, cet escarpement du Jura est peut-être celui qui évolue le plus rapidement de nos jours.

Pendant la période allant de 1973 à 1975 nous avons mesuré (PANCZA 1979) un recul général de $1,7 \mathrm{~mm}$ par an dans les zones gélives de la paroi, ce qui est exceptionnellement élevé. C'est précisément cette intense gélivation qui nous a conduit à multiplier, à préciser et à étendre nos mesures sur une période plus longue, afin de mieux comprendre les causes d'une telle évolution. Ce sont les premiers résultats de ces nouvelles investigations que nous présentons ici.

\section{Site et situation:}

La paroi rocheuse de Château Cugny est généralement subverticale d'une quinzaine de $\mathrm{m}$ de hauteur: étant irrégulière cette hauteur atteint environ $35 \mathrm{~m}$ dans la zone centrale de l'escarpement. L'orientation générale des parois est $\mathrm{S}, \mathrm{SW}$.

Les escarpements de Château Cugny sont taillés dans un calcaire (du Rauracien) crayeux, blanchâtre et à cassure mate. On y rencontre une riche faune de Térébratules. Les couches y sont d'une épaisseur variable, sub-horizontales légèrement inclinées au $\mathrm{N}$.

Le site de Château Cugny ne ressemble pas à celui des autres parois rocheuses du Jura. Généralement, les escarpements de la chaîne jurassienne sont constitués de calcaire dur, massif. Rectilignes ou arquées, ces parois surmontent des éboulis plus ou moins colonisés par la végétation. Cette image ne s'applique pas à Château Cugny où il n'y a pas seulement une paroi mais deux, voire trois, séparées par de profondes échancrures (fig. 1). L'ensemble se présente comme si des zones plus résistantes avaient été entourées de zones plus sensibles à l'érosion: l'action de cette dernière aurait isolé des sortes de "nounatacks» ou "châteaux» actuellement séparés de la paroi principale par des écorchures profondes.' Tout ceci forme un enchevêtrement de parois, de couloirs, de zones éboulées, de corniches et de chicanes assez inextricables (fig. 2).

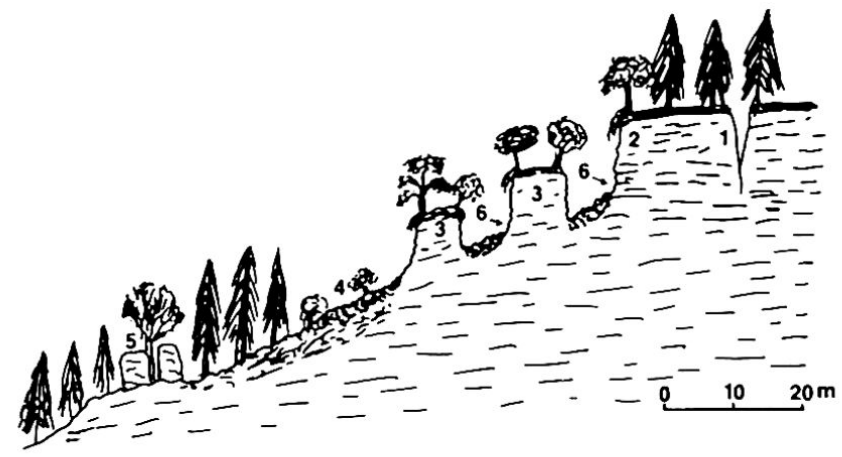

Figure 1 Le Château Cugny: coupe schématique des lieux. 1. échancrure profonde, 2. paroi principale avec racines d'arbres pendant dans le vide, 3. "châteaux", 4. éboulis, 5. gros blocs éboulés, 6. localisation des mesures.

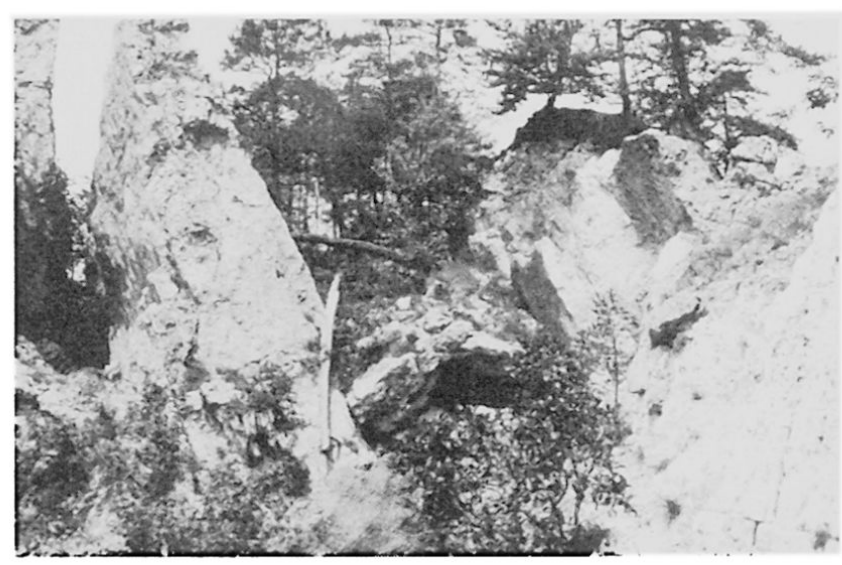

Figure 2 Vue générale des lieux. Blocs éboulés, basculés et falaises en pleine évolution.

André Pancza, Prof., Promenade 1, 2056 Dombresson 


\section{Qualités physiques de la roche:}

Le calcaire de Château Cugny est d'une dureté moyenne (résistance à l'écrasement: 280 à $600 \mathrm{~kg} / \mathrm{cm}^{2}$ ) souvent lézardé de fissures et microfissures. Sa porosité est de 10 à $12 \%$. La courbe de répartition de la dimension des pores (fig. 3), (mesurée à l'aide d'un porosimètre à mercure), indique un fort pourcentage de porosité situé entre 0,1 et $0,5 \mu$. Par des essais de gélifraction en laboratoire, il a été démontré Lautridou (1972) que ce sont précisément les assises ayant une porosité fine, située entre 0,1 et 1 micron, qui sont les plus sensibles à l'action du gel. Les essais au laboratoire de cryoclastie effectués à Caen (PANCZA 1979), montrent que l'évolution du calcaire de Château Cugny est aussi rapide en gélivation expérimentale que dans son milieu naturel. ${ }^{2}$

Ce sont sans doute les qualités physiques de la roche: soit, une forte densité de fissuration ${ }^{3}$ et une porosité élevée qui expliquent la très grande sensibilité au gel de ces assises. ${ }^{4}$

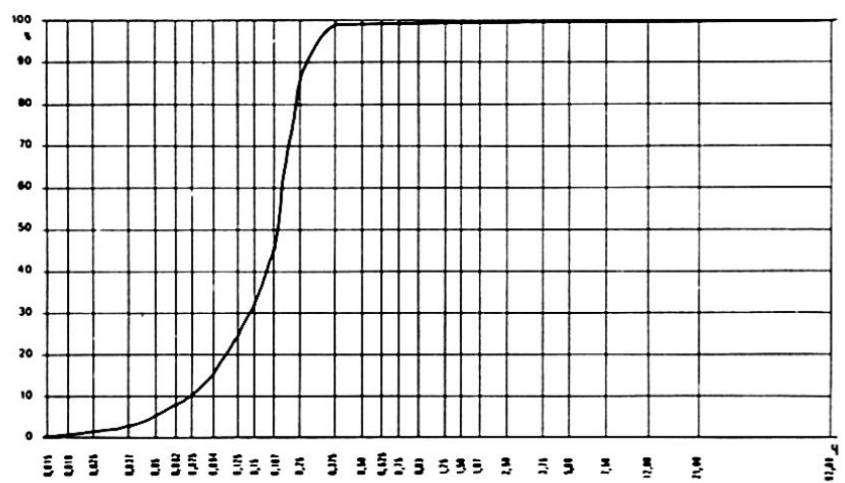

Figure 3 Calcaire crayeux du Château Cugny: courbe de répartition du rayon des pores.

\section{Mise en place et description des installations:}

Un potentiomètre à voies multiples ${ }^{5}$ permettant l'enregistrement de la variation de la température en surface et à diverses profondeurs au sein même de la paroi, a été mis en place l'été 1981 . Ces enregistrements nous renseignent d'une part, sur le nombre de cycles de gel-dégel à diverses profondeurs dans la paroi et d'autre part, sur l'intensité du réchauffement et sur le gradient thermique estival. Un pluviographe installé en 1981, $150 \mathrm{~m}$ plus bas (au lieu dit "Sur le Rang» près de la ferme de la famille Stüssi) fournit des renseignements sur la fréquence et l'intensité des précipitations. Il permet, d'une manière indirecte, d'avoir une idée de la teneur en eau de la roche.

En 1981 nous avons procédé à la mise en place des "pièges à gélifracts». Il s'agit d'installations simples (fig. 4 et 5) appliquées au pied des parois et qui servent à recueillir les éclats détachés.

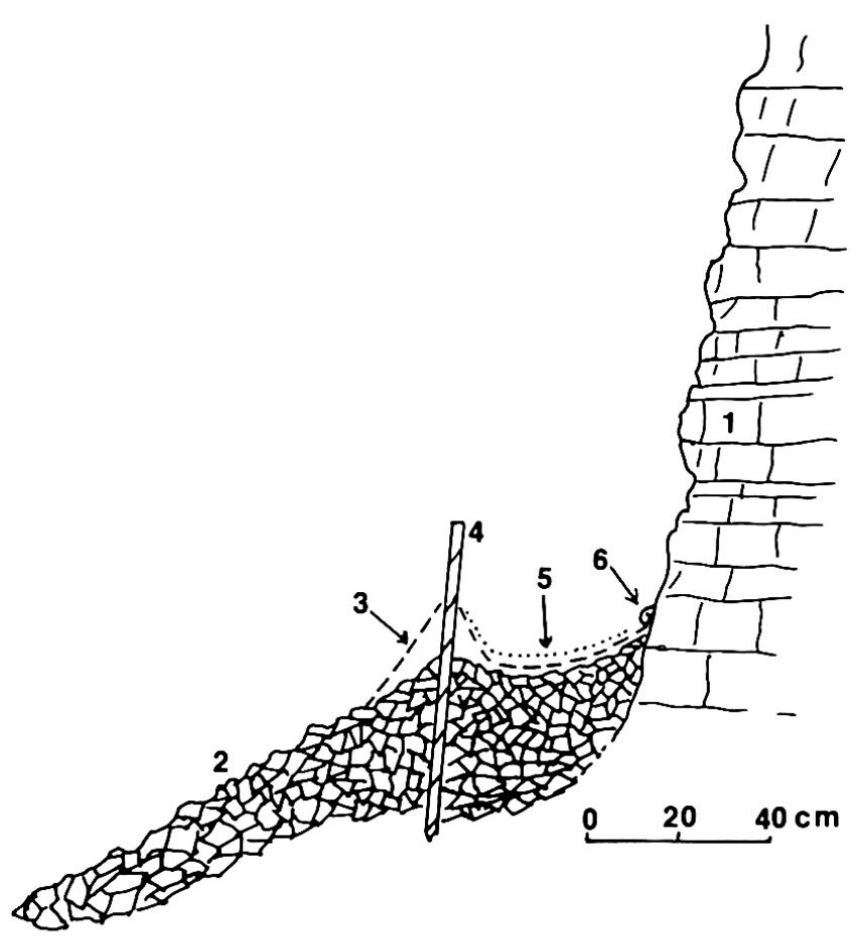

Figure 4 Présentation schématique de l'emplacement des "pièges à gélifracts": 1. paroi rocheuse, 2. éboulis, 3. grillage métallique de $20 \mathrm{~mm}, 4$. fer à bèton de $10 \mathrm{~mm}, 5$. grillage en matière plastique de $4 \mathrm{~mm}, 6$. ciment.

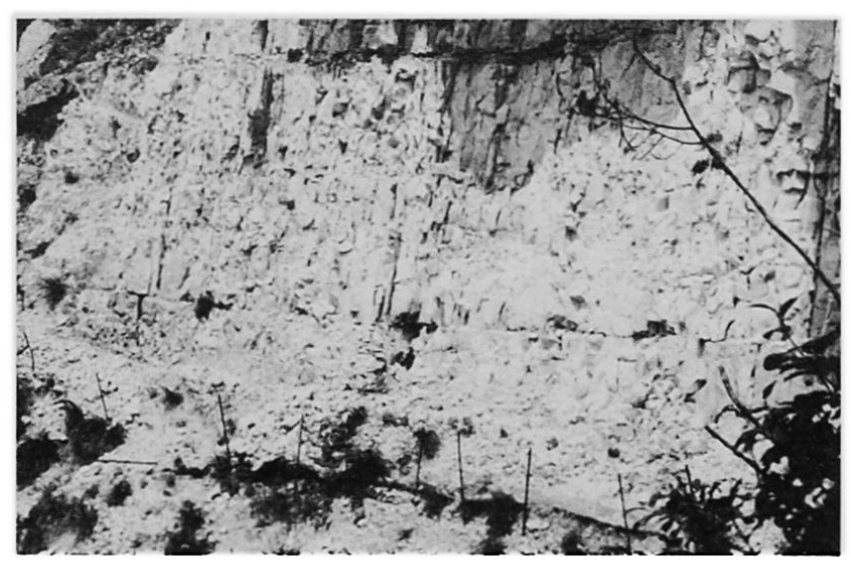

Figure 5 Paroi rocheuse équipée de "pièges à gélifracts", station Ila, vue partielle. La zone centrale a été emportée par un éboulement (photo février 1983).

\section{Description des zones étudiées:}

Les trois premiers pièges (stations Ia, IIa et IIIa) d'un accès facile servent à recueillir et à mesurer la quantité d'éclats détachés chaque mois, tandis que les trois autres (stations Ib, IIb et IIIb) sont considérés comme "totalisateurs" et ne sont vidés que 2 fois par an. Aussi, ces "totalisateurs» ne fournissent-ils que des valeurs saisonnières. 
La station Ia: est située au pied d'un escarpement de $2,6 \mathrm{~m}$ de haut, tourné au $\mathrm{S}$. La longueur du "piège» n'est que de $1,8 \mathrm{~m}$. Cette station sert à tester une zone très gélive qui évolue en forme de niche. Les matériaux recueillis sont assez fins (2-6 cm de longueur).

La station IIa: permet de mesurer l'évolution d'une plus grande surface. Ici, la paroi mesure $7 \mathrm{~m}$ de haut; elle regarde aussi au $\mathrm{S}$. La longueur du "piège» est de $8,5 \mathrm{~m}$ et les éclats qu'on y recueille sont généralement plus gros que dans la zone Ia (4 à $12 \mathrm{~cm}$ de longueur).

La station IIIa: est d'une dimension moyenne. L'escarpement haut de $4 \mathrm{~m}$ est tourné vers l'ouest. Le "piège» long de $5 \mathrm{~m}$ recueille des éclats moyens (3-8 cm).

Les stations Ib, IIb et IIIb: situées à l'E des précédentes, elles sont d'un accès plus difficile. Les escarpements hauts de 2 à $4 \mathrm{~m}$ évoluent très rapidement en fournissant des éclats grossiers et abondants.

En plus des six stations installées et régulièrement surveillées, nous avons pu faire aussi quelques mesures au pied d'une paroi rocheuse pourvue de grillage par les Ponts et chaussées et située en bordure de la route Les Pommerats-Goumois. Cette paroi, haute d'une vingtaine de mètres, est tournée vers l'ouest. Elle est constituée d'une roche aussi gélive que celle de Château Cugny. Les éclats tombés n'étant évacués qu'une fois par an (au printemps), il nous a été possible, grâce à la compréhension des cantonniers, de faire des comparaisons intéressantes avec les mesures effectuées à Château Cugny.

\section{L'enregistrement des températures:}

Les mesures de la température de la roche nous renseignent sur la pénétration du gel au sein de la masse rocheuse. Elles permettent de connaître également le nombre de cycles de gel-dégel à diverses profondeurs. A Château Cugny, dans un escarpement orienté au $\mathrm{S}$, il y a en moyenne 41 gélivations par année en surface, 36 à $6 \mathrm{~cm}, 30$ à $12 \mathrm{~cm}, 18$ à $25 \mathrm{~cm}, 4$ à $50 \mathrm{~cm}$ et seulement 1 à $1 \mathrm{~m}$ de profondeur dans les conditions climatiques actuelles. Le nombre de cycles de gel-dégel est sensiblement plus faible dans une autre orientation: 12 seulement à $25 \mathrm{~cm}$ de profondeur dans une paroi orientée au N.

\section{Régime des chutes de pierres:}

Lors de visites mensuelles, nous avons soigneusement assemblé et pesé les éclats recueillis dans les pièges. C'est aussi pendant ces tournées que des événements particuliers (éboulements, glissements) ont pu être constatés.

\section{Le régime annuel:}

Les figures 6 et 7 montrent le régime des chutes de pierres à Château Cugny. Ce régime (semblable à celui d'un cours d'eau) a été établi grâce à des mesures faites à intervalles réguliers. La période d'observation s'étend sur 22 mois et va de septembre 1981 à juillet 1983. Une observation attentive de ces figures suggère les remarques suivantes:

a) L'intensité des chutes de pierres varie selon les saisons et selon la zone observée. Bien qu'il s'agisse dans chaque section de parois très gélives, le recul n'est pas le même dans chaque zone: il est en moyenne de $3,5 \mathrm{~mm}$ par an au sect. Ia, de $1,7 \mathrm{~mm}$ par an au IIa et $1,2 \mathrm{~mm}$ par an au IIIa.

La zone Ia ne saurait être considérée comme représentative de l'évolution générale; il s'agit là, plutôt d'un maximum temporaire d'une surface restreinte particulièr-ment active évoluant en forme de niche. L'évolution mesurée en IIa, au contraire, donne une idée juste du recul d'une zone gélive tournée au Sud. ${ }^{6} \mathrm{La}$ valeur obtenue correspond d'ailleurs à celle $(1,74 \mathrm{~mm} / \mathrm{an})$ que nous avons enregistrée par la méthode de coloration (1974-75), ceci dans une autre zone semblable tournée également au Sud.

Le recul de la section IIIa $(1,2 \mathrm{~mm})$ reflète fidèlement l'évolution d'une paroi gélive orientée à l'Est ou à l'Ouest.

RAPP (1960), RUDBERG (1960) et leurs élèves ont mesuré l'action du gel dans les parois rocheuses en Suède. D'une manière générale, les valeurs obtenues par les Suédois $(0,5 \mathrm{~mm}$ en calcaire, $0,7 \mathrm{~mm}$ en granite, $0,9 \mathrm{~mm}$ en dolomite) sont plus faibles que celles de Château Cugny. POSER (1954) estime le recul des parois de gneiss, schiste et serpentine à 0,7-1,0 mm/an dans les Alpes autrichiennes. KING (1956) a mesuré une évolution de $1,5 \mathrm{~mm}$ en Afrique du Sud.

b) Durant l'été, la chute des pierres est 10 à 20 fois plus faible qu'en hiver. Pendant l'hiver, d'une manière générale, le détachement des éclats est provoqué par le gel; aussi, les débris sont-ils répartis uniformément le long des «pièges». En été, on constate une inégale répartition des éclats détachés: seules certaines zones fournissent des débris sous forme de petits éboulements locaux. De tels éboulements se sont produits pendant les mois de juin et août 1982 dans la section IIIa et en février 1983 en IIa. ${ }^{7}$

Il est difficile de connaître la cause exacte de l'évolution estivale des parois de Château Cugny. Ces détachements de pierres peuvent avoir des causes multiples parmi lesquelles la thermoclastie et les périodes d'humectation et de dessication sont les plus importantes. Ces facteurs physiques ne font peut-étre que compléter l'action fondamentale 
Figure 6 Régime des chutes de pierres à Château Cugny

\begin{tabular}{|c|c|c|c|c|c|c|c|c|c|}
\hline \multirow[b]{2}{*}{ Date } & \multirow[b]{2}{*}{$\begin{array}{l}\text { Durée } \\
\text { (jours) }\end{array}$} & \multicolumn{3}{|c|}{$\begin{array}{l}\quad 1981-1982 \\
\text { Poids des éclats en kg }\end{array}$} & \multirow[b]{2}{*}{ Date } & \multirow[b]{2}{*}{$\begin{array}{l}\text { Durée } \\
\text { (jours) }\end{array}$} & \multicolumn{3}{|c|}{$\begin{array}{l}1982-83 \\
\text { Poids des èclats en } \mathrm{kg}\end{array}$} \\
\hline & & $\begin{array}{l}\text { Sect. la } \\
\left(4,7 \mathrm{~m}^{2}\right)\end{array}$ & $\begin{array}{l}\text { Sect. lla } \\
\left(60 \mathrm{~m}^{2}\right)\end{array}$ & $\begin{array}{l}\text { Sect. Illa } \\
\left(18 \mathrm{~m}^{2}\right)\end{array}$ & & & $\begin{array}{l}\text { Sect. la } \\
\left(4,7 \mathrm{~m}^{2}\right)\end{array}$ & $\begin{array}{l}\text { Sect. lla } \\
\left(60 \mathrm{~m}^{2}\right)\end{array}$ & $\begin{array}{l}\text { Sect. Illa } \\
\left(18 \mathrm{~m}^{2}\right)\end{array}$ \\
\hline 20 sept. & & & & & 22 août & & & & \\
\hline & 31 & 2,1 & 7.1 & 2,1 & & 30 & 0,2 & 1,6 & 0,4 \\
\hline 2100. & 25 & 3,4 & 14,0 & 4,2 & & 29 & 0,1 & 0,8 & 06 \\
\hline 15 nov. & & & & & 20 oct. & & & & \\
\hline 17 déc. & 32 & 11.1 & 51,0 & 15,0 & 14 nov. & 25 & 0,7 & 0,9 & 0,7 \\
\hline & 31 & 21,0 & 58.0 & 11,0 & & 35 & 3,4 & 12,0 & 1,2 \\
\hline 15 janv. & 33 & 10,5 & 64,0 & 7,1 & 19 déc. & 29 & 6,0 & 65,0 & 8,1 \\
\hline 18 fév. & 28 & 5,1 & 5,3 & 5,8 & & 37 & 6.2 & $130.0 !$ & 154 \\
\hline 18 mars & & & & & 23 fév. & & & & \\
\hline 20 av. & 33 & 1,1 & 1,2 & $3,3 !$ & 20 mars & 25 & 8,2 & 87,0 & 15,0 \\
\hline 20 mai & 30 & 0,2 & 2,1 & 0,3 & 22 av. & 33 & 8,0 & 41,0 & 4,0 \\
\hline 18 juin & 31 & 0,5 & 1,7 & 24,0 & 30 mai & 38 & 1,8 & 13,0 & 2,7 \\
\hline 20 juill. & 32 & 1,2 & 3,8 & 2,0 & 10 juill. & 41 & 1,4 & 4,0 & 0,8 \\
\hline 22 août & 33 & $\begin{array}{r}1,0 \\
57,2\end{array}$ & $\frac{1,4}{209,6}$ & $\begin{array}{r}3,6 \\
77,8\end{array}$ & & & $\overline{36,0}$ & $\overline{355,0}$ & $\overline{48,9}$ \\
\hline
\end{tabular}

du gel hivernal: le gel aurait fracturé, miné la roche et la thermoclastie ou les pluies intenses ne feraient qu'achever l'action en provoquant le détachement des éclats. RAPP (1960), HEIM (1932), JÖRSTAD (1956) insistent sur le rôle des pluies dans l'évolution des surfaces rocheuses, tandis que ALIMEN (1958), BRADLEY (1963), COOKE (1970) et autres mettent en évidence le rôle de la thermoclastie.

c) La quantité de pierres tombées pendant les mois d'hiver est certes proportionnelle au nombre de cycles de gel-dégel, mais d'une manière générale la teneur en humidité de la roche (gel précédé de pluie ou de fusion de la neige) est peut-être un facteur plus important que l'intensité même de la gélivation. Mais seules des observations fréquentes (tous les 10 jours ou une fois par semaine) pourraient renseigner avec précision sur le rôle de l'humectation de la roche.

\section{Le régime saisonnier:}

La quantité d'éclats recueillis dans les «totalisateurs» (sections Ib, IIb et IIIb) et pesés deux fois par an (avril et octobre) confirme d'une part, le recul rapide des parois à Château Cugny et d'autre part, la variation saisonnière des chutes de pierres déjà constatée. Ce secteur est aussi le siège d'éboulements locaux: preuve en est, la partie centrale du piège IIb démolie par un éboulement durant l'été 1982.
Les pierres recueillies dans les «totalisateurs» en bordure de la route Les Pommerats-Goumois correspondent à un recul d'environ $1,9 \mathrm{~mm}$. Cette paroi est orientée à l'ouest et on y observe des suintements locaux qui y entretiennent une humectation régulière.

\section{Le régime journalier:}

L'idée de contrôler la fréquence des chutes de pierres d'une manière continue nous est venue lors d'une de nos visites mensuelles, par une matinée ensoleillée précédée d'une nuit froide où la température de l'air est descendue à $-10^{\circ} \mathrm{C}$. Pendant les heures de dégel, nous avons remarqué un grand nombre de bruits caractéristiques de chutes de pierres sur l'éboulis.

Ces bruits étaient suffisamment audibles pour être enregistrés sur une bande magnétique, à condition de disposer d'un enregistreur sensible muni d'un microphone orientable. Nous avons procédé à ces enregistrements les 24 et 25 janvier et les 22 et 23 février 1983. Les figures $9 \mathrm{~A}$ et B montrent en même temps: les chutes de pierres et l'évolution de la température de l'air ainsi que celle de la roche. La température fut assez semblable durant les 2 nuits avec de faibles gelées de -5 à $-6^{\circ} \mathrm{C}$. En revanche, le réchauffement diurne fut sensiblement différent. Tandis que le 25 janvier était nuageux avec seulement quelques éclaircies, le 23 février fut particulièrement clair et ensoleillé. Nous sommes donc en présence de 2 phases de 


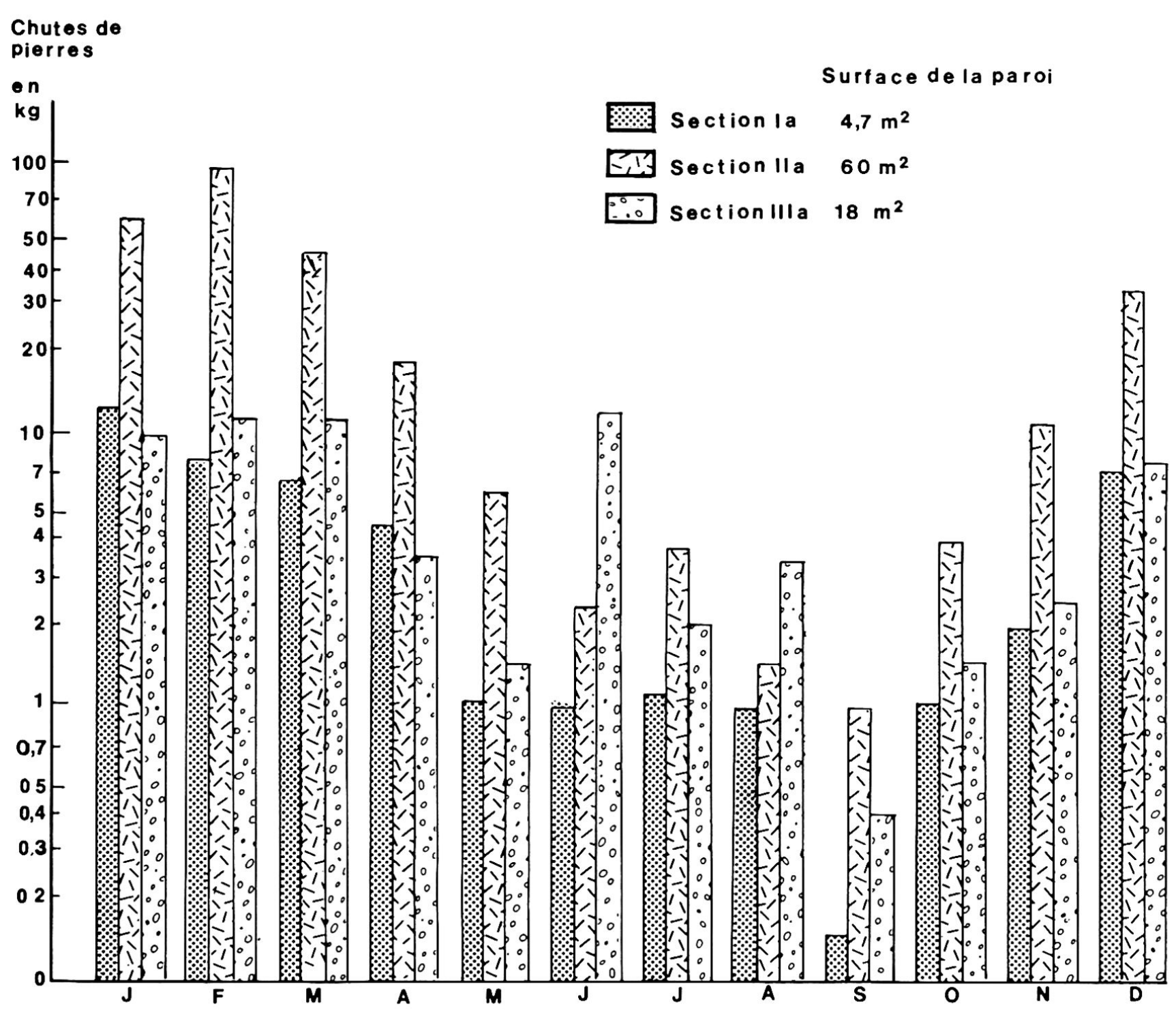

Figure 7 Régime des chutes de pierres à Château Cugny (sept. 81-juill. 83), moyenne arithmétique des deux ans.

gel sensiblement identiques suivies de dégels différents. La comparaison des enregistrements nous montre que:

a) Les pierres tombent très fréquemment pendant les premières heures de dégel surtout si le soleil provoque un brusque réchauffement de la surface rocheuse. Ensuite, au . cours de l'après-midi, le nombre de chutes diminue.

b) Durant la gélivation (nuit) les chutes de pierres sont très rares.

c) Pendant un jour nuageux avec éclaircies, la période des chutes de pierres est plus longue, moins concentrée que lors d'un jour ensoleillé. Est-ce également le cas dans une paroi tournée au nord?

d) Le nombre de chutes est plus élevé pendant un jour ensoleillé $(2 \mathrm{x}$ plus de chutes le 23 février que le 25 janvier).
Ce dernier fait est surprenant: il est probable que le brusque réchauffement accélère le détachement des pierres déjà déchaussées.

La fréquence élevée des chutes de pierres pendant les cycles journaliers de gel-dégel fut également constatée par l'observation indirecte suivante faite le 23 février 1983. Il fut frappant de voir ce jour-là le nombre de traces de chutes de pierres sur la surface neigeuse. Il n'avait pas neigé depuis huit jours et seules certaines zones de l'éboulis, situées à l'ombre, portaient encore une couche de neige résiduelle. Dans l'une des zones, proche d'une paroi gélive, nous avons dénombré 36 traces de chutes ou de déplacements d'éclats au $\mathrm{m}^{2}$. Ayant rassemblé et pesé les 36 pierres, on obtint un poids total de 2,8 kg: même s'il s'agit d'un maximum, il est peu commun de constater une telle alimentation d'éboulis en huit jours. 


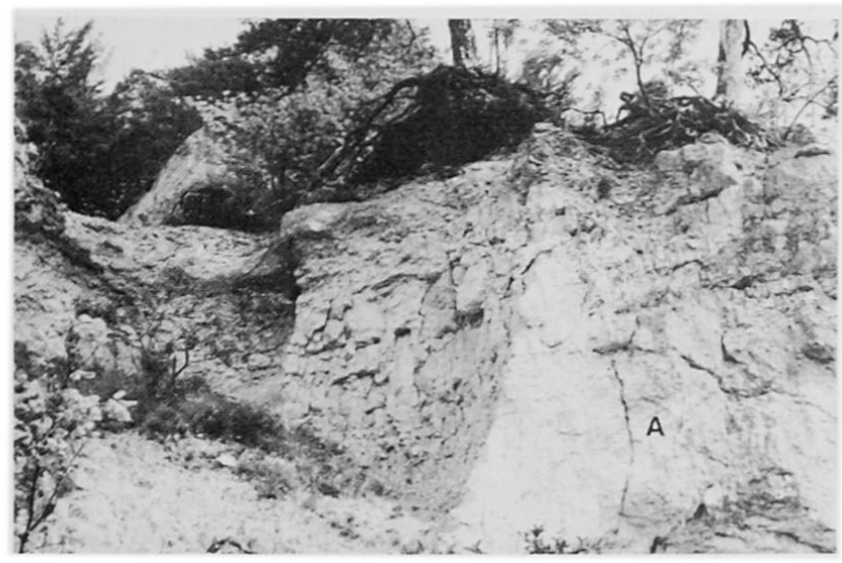

Figure 8 Le Château Cugny, vue partielle de l'un des escarpements. On remarque les racines d'arbres pendant dans le vide et une zone $(A)$ de futur éboulement (photo oct. 1982).

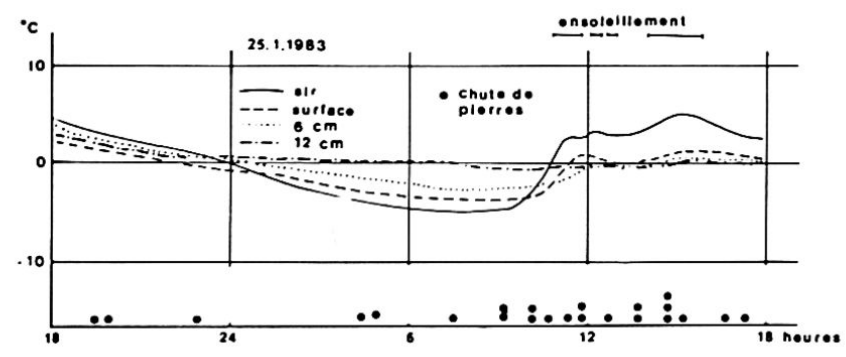

Figure 9A Variations de la température de la roche et chutes de pierres. Régime journalier des chutes de pierres lors d'une faible gelée suivie d'un dégel superficiel.

\section{Les éboulements:}

A Château Cugny les éboulements sont rares mais non exceptionnels. Leur contribution au recul de la paroi est réelle. Pendant les quatre dernières années il s'est produit trois éboulements dans les parois surveillées, déplaçant au total environ $3 \mathrm{~m}^{3}$ de roche. Ce sont généralement des zones surplombant des abris sousroche qui, déséquilibrées, se mettent brusquement en mouvement. Il existe à Château Cugny une sorte d'équilibre fait de constants réajustements entre l'action du gel provoquant le détachement des éclats isolés et les éboulements: la gélivation prépare en quelque sorte ces derniers.

En observant les lieux, on remarque aisément des traces d'éboulements récents et d'autres plus anciens. Il y a aussi les éboulements qui se préparent: l'écartement progressif de certaines fissures, les traces de glissements récents en sont des signes précurseurs.

Ce sont des éboulements anciens, probablement de la période Würmienne, qui ont déplacé la plus grande masse de roche et aussi les plus gros blocs. Ceux-ci une fois en mouvement ont parcouru plusieurs centaines de mètres avant de s'immobiliser à mipente, sur une zone peu inclinée actuellement recouverte par la forêt. Leur grand nombre et leur taille (les plus gros atteignent $100 \mathrm{~m}^{3}$ ) prouvent l'importance de ces mouvements de masse.

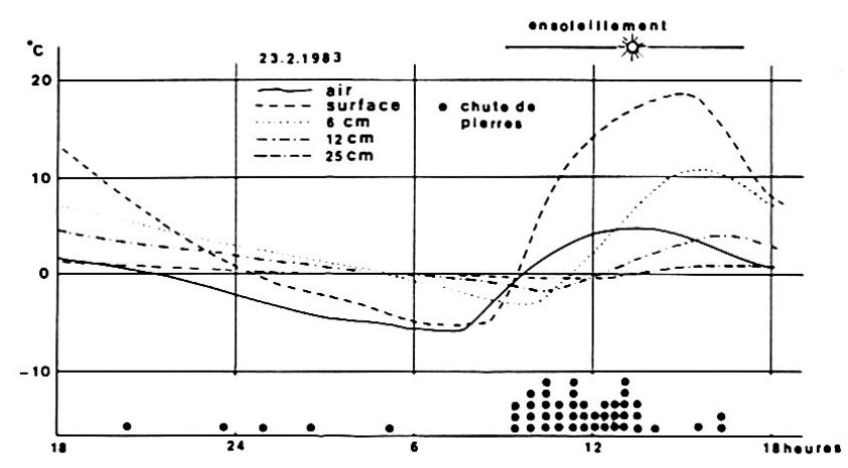

Figure 9B Variations de la température de la roche et des chutes de pierres lors de faibles gelées nocturnes suivies d'intense réchauffement.

La dimension des blocs éboulés récemment est plus modeste (généralement inférieure au $\mathrm{m}^{3}$ ). Leur élan initial ne les transporte guère au-delà de la limite inférieure de l'éboulis, où ils sont généralement bloqués par les arbres de la forêt.

\section{Conclusion:}

Château Cugny est un endroit privilégié où l'évolution actuelle des parois rocheuses est particulièrement rapide. L'action du gel y détache chaque année des masses considérables de gélifracts, ce qui permet, à l'aide d'installations simples, de mesurer le régime des chutes de pierres.

Les deux exemples de cycles de gel-dégel journaliers montrent la variation des chutes de pierres au cours de la journée, ceci en fonction de la température.

Les fréquents éboulements de masses rocheuses contribuent aussi au recul rapide des parois de Château Cugny. Ces éboulements se produisent en alternance avec l'action de la gélivation: c'est le recul rapide des zones gélives du pied de l'escarpement qui provoque l'éboulement périodique des zones susjacentes moins sensibles au gel.

\section{Zusammenfassung}

"Château Cugny» ist eine außergewöhnliche Lokalität, die sich durch eine besonders hohe Erosionsrate der Felswände auszeichnet. Im Verlaufe eines Jahres wird durch den Frost so viel Steinschlag verursacht, daß es mit einfachen Mitteln möglich ist, jahreszeitliche Schwankungen des «Erosions»prozesses zu messen.

Die zwei Beispiele der Frosttageszyklen zeigen die Änderung der Steinschlaghäufigkeit in Abhängigkeit der Tageszeit und der Temperatur. Das häufige Ausbrechen der frostempfindlichen Formationen verursacht periodische lokale Bergstürze des überliegenden, frostunempfindlichen Gesteins. 


\section{Abstract}

Château Cugny is an exceptional place where the actual cliff's evolution is quite rapid. Because of the frost action, considerable quantities of frost-rived rubble broke off the cliffs each year. The relative frequency of rock falls enables measurements to be made with simple installations. Two examples of the daily freeze-thaw cycle show the variations of the rock falls during the day according to the temperature's changes. Frequent rock falls contribute to Château Cugny's cliffs rapid scarp retreat.

These rock falls occur by a 2-stage process: first the frost susceptible lower portion of the cliff breaks apart resulting in undermining of the overlying non frostsusceptible portion which subsequently breaks off and falls down.

\section{Remerciements:}

Nous tenons à remercier très chaleureusement Monsieur F. Stüssi d'avoir bien voulu s'occuper du pluviographe de "Sur le Rang» et de nous avoir ainsi fourni de précieux renseignements. Nous remercions également Messieurs C. Béguin et J.-P. Launaz, nos amis, qui nous ont aidé lors de l'installation des appareils. Notre gratitude va aussi au Centre de Géomorphologie du CNRS de Caen et au Centre d'hydrogéologie de l'Université de Neuchâtel pour avoir mis à notre disposition un potentiomètre et un pluviographe. Enfin, nous remercions le Fonds national suisse de la recherche scientifique pour son soutien financier.

\section{Notes:}

'Des échancrures situées sur le plateau sommital à une trentaine de $\mathrm{m}$ de la paroi principale prouvent que cette évolution se poursuit. On y observe l'ablation du calcaire et sa fragmentation par le gel.

${ }^{2}$ Sur les 6 échantillons provenant de Château Cugny et soumis à la gélivation expérimentale 3 ont éclaté et les 3 autres ont fourni entre 6 et $30 \%$ de débris.

${ }^{3}$ La densité de fissuration des zones gélives est de 3,4 en moyenne (distance moyenne entre 2 fissures consécutives en $\mathrm{cm})$.

${ }^{4}$ Les surfaces témoins coloriées en 1973, Pancza (1979, p. 125) ont été intégralement détachées en 10 ans. II n'y a donc plus de trace de peinture sur la paroi; par contre, on retrouve des éclats détachés et portant des taches de couleur à la surface de l'éboulis.

${ }^{5}$ |l s'agit d'un appareil de marque Grant à 9 voies, alimenté par piles et permettant des enregistrements à intervalles réguliers (toutes les $2 \mathrm{~h}$ ). La précision des mesures est de $\pm 0,5^{\circ} \mathrm{C}$. Les thermosondes sont placées respectivement à $6,12,25,50$ et $100 \mathrm{~cm}$ de profondeur dans une paroi tournée au S-SE. La température de l'air est également enregistrée. Deux autres thermosondes permettent l'enregistrement de la température à $25 \mathrm{~cm}$ de profondeur dans deux parois orientées à l'E et au $\mathrm{N}-\mathrm{NE}$.

${ }^{6}$ Cette évolution rapide est visible aussi par la position en corniche des pins sylvestres dont les racines pendent dans le vide (fig. 8). La fraîcheur des èclats dans l'éboulis en est aussi une preuve.

${ }^{7}$ Les chutes importantes enregistrées dans la station lla pendant les mois suivant l'éboulement sont à attribuer au rééquilibre de la surface rocheuse.

\section{Bibliographie}

ALIMEN M. H. (1958): Premiers résultats de l'étude morphométrique de cailloutis sahariens, Rev. Geom. Dyn. IX ${ }^{\mathrm{e}}$ année, pp. 161-173.

BRADLEY W.C. (1963): Large-scale exfoliation in massive sandstones of the Colorado Plateau, Bull. Geol. Am. Soc., 74, pp. 519-528

COOKE R. U. (1970): Stones pavements in deserts, Ann. Ass. Geogr. No. 60, pp. 560-577.

COUTARD J.-P., PANCZA A. (1981): Expèrience de thermoclastie sur quelques calcaires jurassiens, Geogr. Helv. 1, pp. 9-13.

HEIM A. (1932): Bergsturz und Menschenleben, Vierteljahrsschr. d. Nat. forsch. Ges. Zurich, 77.

JÖRSTAD F. A. (1956): Fjellskredet ved Tjelle, et 200-ars minne. "Naturen". Bergen.

KING L.C. (1956): Research on slopes in South Africa, Prem. Rapp. de la Comm. pour l'Et. des Versants, I.G.U. Amsterdam.

LAUTRIDOU J.-P. (1972): Bilan des recherches de gélifraction expérimentale effectuées au Centre de géomorphologie. Bull. Centre géom. 13-14-15, pp. 65-73.

PANCZA A. (1979): Contribution à l'étude des formations périglaciaires dans le Jura, thèse, Univ. Neuchâtel, 187 p.

POSER H. (1954): Die Periglazial-Erscheinungen in der Umgebung der Gletscher des Zemmgrundes, Göttinger Geogr. Abh., 15:Il.

RAPP A. (1960): Recent development of mountain slopes in Kärkevagge and surroundings, Northern Scandinavia, Geogr. Ann. Univ. Uppsala, XLII, pp. 72-200.

RAPP A., RUDBERG S. (1960): Recent periglacial phenomena in Sweden, Biul. Perygl. No. 8, pp. 143-154.

RAPP A. (1961): Studies of the postglacial development of mountain slopes, Uppsala Univ. Geogr. Instit. Ser. A, Nr. 159, $11 \mathrm{p}$. 